\title{
The Effect of Sleepaway Camp Attendance on College Homesickness
}

\author{
Liora Tuchman $^{1}$ and Ida Williams ${ }^{1}$ \\ ${ }^{1}$ Western High School, Davie, FL, USA
}

\section{ABSTRACT}

The purpose of this study was to examine if going to sleepaway camp before the age of 16 was a factor that reduced levels of homesickness in first-year students at the University of Florida. In this study, the researcher sent out an online survey to first-year students at the University of Florida through social media, email, and text message. The questions asked the participants about both college and camp experience and asked the participants if they attended sleepaway camp before the age of 16. The results show going to sleepaway camp had a minimal effect on homesickness levels in first-year students at the University of Florida, but attending sleepaway camp had a positive impact on "college experience." These findings provide implications for future research by showing factors of the camp experience that help in the college experience. Ultimately, discovering if there is a connection in attending sleepaway camp and a positive camp experience will not only help the students themselves but also the camp community and parents of children who attend sleepaway camp.

\section{Introduction}

Summer camps were established in the late 19th century as a place for young children, ages eight to sixteen, to go during their summer break to enjoy activities in nature and spend time with friends (Petrzela, 2018). Summer camps can be broken down into two types: day camps, where children go for the day and return home at night, or sleepaway camp, where children stay overnight for as little as three days or as long as eight weeks (Broussard \& Meaux, 2007). At sleepaway camps, children live in a cabin with up to twelve others and must share bathrooms, shelf space, and even eat together (Baskin, 2013). Summer camps are diverse, depending on location, size, length, or even specificity. There are gender-specific camps, religious camps, camps for children with medical conditions, and special interest camps, where children focus specifically on their hobbies (Broussard \& Meaux, 2007). Sleepaway camps, specifically, have been found to teach children positive social skills, while also giving them a so-called "break from reality" by not having access to technology, since most sleepaway camps prohibit the use of cell phones or computers (Cohen, 2014; Diamond, 2015). Skills learned at camps may be helpful to children as they enter college and the workplace.

Although various investigators have based their studies on broad social skills learned at different sleepaway camps, the research conducted relates specifically to homesickness in first-year college students, specifically at the University of Florida, and how attending sleepaway camp could be a factor that either increased or decreased their levels of homesickness. For the purpose of this mixed-method study, homesickness will be defined as "longing for home and family while absent from them" (Merriam-Webster's Dictionary, n.d.). This study can influence parents of younger children on their decision of whether to send their children to sleepaway camp. Homesickness in first-year college students is hypothesized to be lower in those who have attended summer camp versus those who have not. This will be discovered through the research question: Is there a correlation between going to sleepaway camp as a child and levels of homesickness in first-year college students at the University of Florida? 


\title{
Literature Review
}

\author{
Social Skills Learned
}

At sleepaway camp, young children, some as young as six years old, join others in a remote environment and can develop some of life's most necessary social skills (Monke, 2018). In a study done by the American Camp Association (2005), surveys done on both campers and parents showed growth in social skills, such as independence and leadership, from pre-camp to post-camp, some skills even being maintained or growing further six months later. Throughout the duration of camp, children live away from their homes and their parents for varying lengths of time. Young children have to do both simple and complex tasks without help or influence from their parents (Cohen, 2014). These tasks promote independence without children even realizing it, which can help them in the future.

At most sleepaway camps, children do not have access to technology, such as their phones or computers, which benefits the child's brain by giving it time to relax, while also letting them live in the moment (Rampton, 2017). Children at camp also learn teamwork. Since many activities are team-based, they learn how to communicate with others respectfully, while also sharing their opinions. These social skills, along with many others, are life skills that are not only beneficial to children during their young life, but during their college and adult lives as well.

\section{Connection with Peers at Camp}

Children build a special relationship with their peers at sleepaway camp, which benefits them for the rest of their lives. Many programs and activities at camp help form friendships that could last a lifetime, not just a summer (Thompson, 2016). These friendships help children at camp feel more included and part of a community. Children bond with their fellow campers over things such as shared experiences or similar hobbies. They also learn how to cooperate and maturely talk to their peers by having to problem-solve and share (Thompson, 2016). Living with many other children for long periods allows them to act as their "normal" self, which can not only help them find friends with similar personalities but can also help them understand themselves (Camp Experts, n.d.). According to a study done by Melzter and Rourke (2010), campers reported they felt closer to their camp friends than their school friends because camp peers have more experiences that they can relate to. Camp friendships help children feel more connected, while also teaching them skills that will help them in their future bonds.

\section{Homesickness in College}

Many students have a hard time dealing with homesickness in college. Around $69 \%$ of first-year college students have reported they suffer from severe homesickness in their first year (Bajrovic, Baker, Markel, Oosting, \& Hollingsworth, n.d.) Whether it is their first time being away from home, or not knowing how to transition, it is hard for many firstyear college students to get acclimated to their new life. Homesickness on the college campus can be shown through different indicators, such as visiting home often, isolating yourself, or struggling to connect with others (Esposito, 2017). These hardships can be dealt with at different phases of college as well (Khademi \& Aghdam, 2013). Many college students have reported they are homesick because they do not feel connected to anyone at their school, or they do not know how to live without their parents (Livingston, 2011). Urani, Miller, Johnson, and Petzel (2003) performed a study to examine the relationship between students with social anxiety and their homesickness levels. Results showed a "positive relationship between social anxiety and homesickness at the beginning of the semester" (p.1). Additionally, the researchers provided explanations of increased social anxiety and increased homesickness at the beginning of the semester, by explaining how many first-year students feel more anxiety at the beginning of the year versus the end. Homesickness may seem inevitable to many college students, but these students can combat it. 


\section{Parallels Between Sleepaway Camp and College}

Whether it is a long-distance from home, having to function on small amounts of sleep, or living with other people, sleepaway camps, and college show many parallels (Olsen, 2018). If children have been to sleepaway camp before they attend college, they are more likely to respond positively to the drastic change in lifestyle between living at home versus living at college. They have already lived without their parents, lived with others, shared communal spaces, and learned how to make new friends. They have already gone through the "transition stage", meaning this change in lifestyle, and know how to immerse themselves in a new environment (Olsen, 2018). Schwartz (2015) explained how going to sleepaway camp helped her in college by making it easier to live with a roommate and sharing spaces with others. Olsen also explained that college requires students to find different buildings and navigate around campus, like going to sleepaway camp for the first time. Although college campuses are more complex than a camp, these students will already be comfortable with navigating through a new setting. The parallels between sleepaway camp and college are something that can be helpful when going to college for the first time.

\section{Method}

\section{Participants and Collection of Data}

The most appropriate method for this study was to conduct a survey. Nearly 100 first-year students at the University of Florida completed this survey in December of 2019. The survey was limited to only first-year students at University Florida to keep the data more concise. The University of Florida was chosen as it was the most accessible university to get participants. A difference in college year could affect the data due to more experience in college and add another variable that the researcher was not looking to find. The researcher's first step was to construct the survey and questionnaire through Google Forms. The survey section included many questions on both college and camp experiences that the students answered through a Likert Scale and multiple-choice questions. A Likert Scale was used to give the participants a broad range of answer choices for certain questions. Specific to this study, the Likert Scale questions included how the respondent would rate their adjustment to college life, living with a roommate, homesickness levels, and camp experience. The multiple-choice questions included the number of times the respondent called home on average during their first semester, how many times they visited home in their first semester, if they attended a sleepaway camp, number of summers attended sleepaway camp if any, and length of the sleepaway camp if attended. According to Espositio (2017), going home often is a sign of homesickness, so that is why a question was asked regarding how many times the student went home during their first semester. The survey ended with one open-ended question that sought student perspectives on the connection between sleepaway camp and college homesickness. One main challenge in conducting this survey was that since it was anonymous, there was no way to make sure that the person replying is a freshman at the University of Florida. The first question in the survey asked if the participant is, in fact, a freshman at the University of Florida, which kept the data reliable.

\section{Technology Used}

To perform this study and interpret the data, the researcher used two primary technology sources. These consisted of Google Forms and Microsoft Excel. The survey, made through Google Forms, was sent out by the researcher through social media, email, and text to students at the University of Florida to attain participants. The researcher then used Microsoft Excel to create the graphs and charts that visually showcased the data. The researcher made a graph and chart for each question and compared the answers from those who went to sleepaway camp and those who did not. 


\section{Method Defense}

A survey was chosen by the researcher to receive the most concise and accurate results, as well as it also allowed the researcher to reach the largest sample size. In contrast to conducting interviews or a case study, a survey permitted the researcher to reach a large number of first-year students at the University of Florida. It was able to be sent out through social media and text messages, as opposed to having to go on campus and ask students in person. A survey also allowed the data to be more concise and easier to analyze, as well as organize into graphs.

\section{Ethical Safeguards}

This survey was completely anonymous, and no personal information was asked of the respondent. There was also a consent form at the beginning of the survey that included the purpose, risks, and benefits of the study and explained that the participant must be 18 years or older to take the survey (see Appendix A). All respondents had to agree that they were 18 years or older to ensure that there were no minors. The respondent was also assured that they would be allowed to skip any question they did not feel comfortable answering, and could stop the survey at any time.

\section{Results}

\section{Eligible Participants}

After randomly sending out the survey to first-year students at the University of Florida, 94 students responded. Fourteen of these responses were discarded due to these participants answering "no" to the first question, which asked, "Are you a first-year student at the University of Florida?" This left the researcher with 80 total responses of first-year students at the University of Florida.

\section{Participant Demographics}

A primary question the participant needed to answer was whether or not they attended sleepaway camp before the age of 16. Figure 1 shows that $42.5 \%$ (34) of first-year students at the University of Florida surveyed have not attended sleepaway camp before the age of 16 . Figure 1 also shows that $57.5 \%$ (46) first-year students at the University of Florida surveyed have attended sleepaway camp before the age of 16 . This question was asked in order to compare the college experiences and homesickness levels of each group.

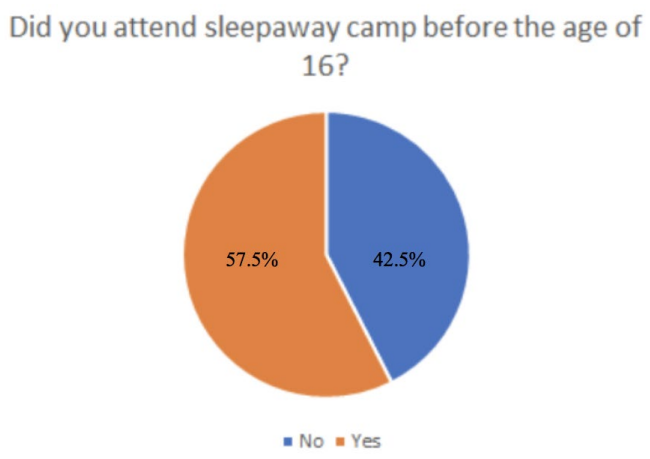

Figure 1. Graph indicating the percentage of participants who attended and did not attend sleepaway camp 


\section{Homesickness in First-Year Students}

The main question the researcher sought to answer related to homesickness levels in the first semester of the participants' first year of college. The survey question asked, "How homesick did you feel during your first semester of college?" A 1 meant very homesick, and a 5 meant not homesick at all. The results of this question did not show a major difference between students who attended sleepaway camp before the age of 16 versus students who did not. (See figure 2) The majority of participants rated their homesickness levels a 4, meaning "not homesick." One slight difference shown is that for participants who rated their homesickness levels a 1, meaning very homesick, 5 out of 7 responses were from participants who did not attend sleepaway camp. This can show that going to sleepaway camp can slightly affect levels of homesickness in first-year college students.

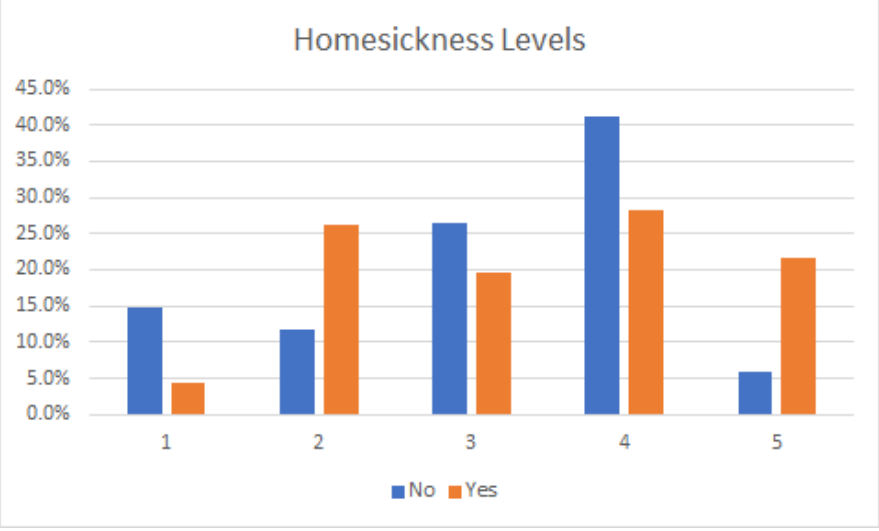

Figure 2. Graph indicating how homesick the participant felt during the first semester.

There are indicators that are signs that a student is feeling homesick in college, such as going home often, isolating yourself, and crying often (Esposito, 2017). Questions asked in the survey sought to find if the student showed any of these signs. Figure 3 displays the difference in how many times the student called home per week between students who have attended sleep away camp and those who have not. The orange bars represent those who have attended sleepaway camp, and the blue bars represent those who have not attended sleepaway camp. The graph shows that, on average, most students call home every day.

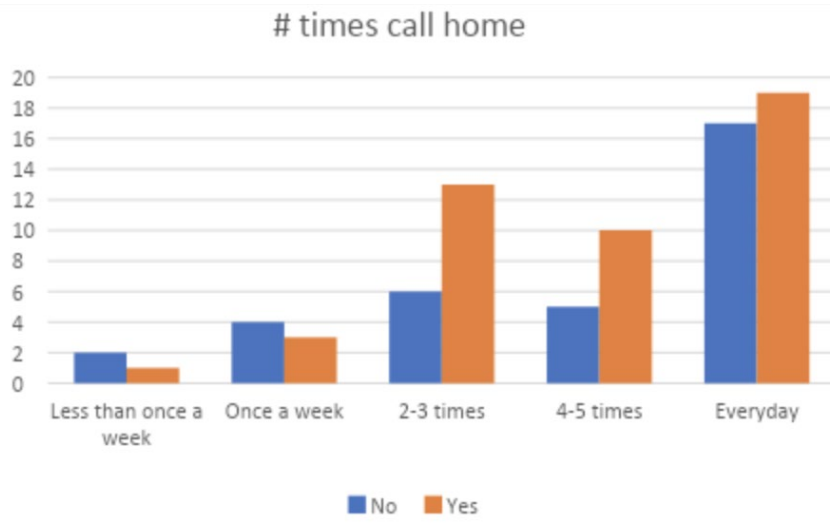

Figure 3. Graph indicating how many times participants called home per week during the first semester

Figure 4 shows the difference in how many times the student visited home on average during their first semester. As Esposito (2017) mentions in her list of signs of homesickness in college students, visiting home often is a large 
indicator of homesickness. Most students, both those who have and have not attended sleepaway camp, visited home 1-3 times in their first semester. This question does not show a major difference between those who have and have not been to sleepaway camp. This survey did not ask if students live in state or out, which could impact the ease of traveling home during the semester.

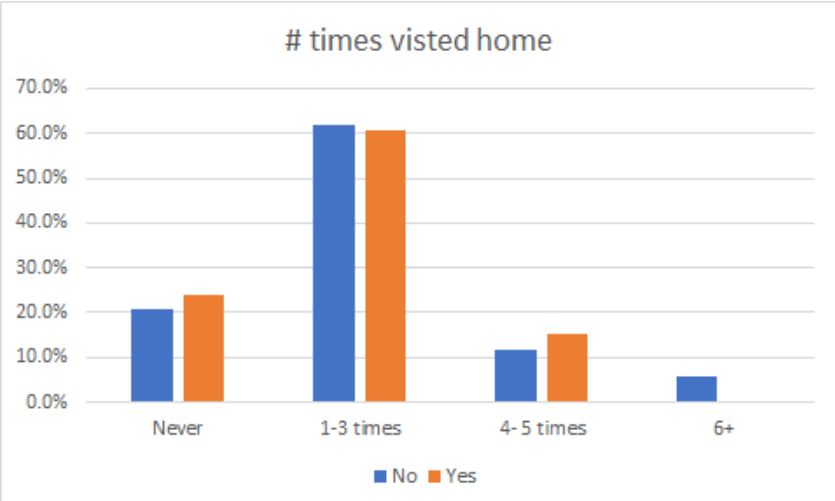

Figure 4. Graph indicating how many times the participant visited home during the first semester

\section{College Experience}

The researcher sought to collect answers to various questions relating to adjustment to the college experience such as living with a roommate. Olsen (2015) explained that there are many parallels between sleepaway camp and college, many that could positively affect the college experience. One survey question asked the students about how well they felt they adjusted to college life in their first semester. The students answered based on a Likert Scale, a 1 being not well and a 5 being very well. Most students choose between a 3 and 4, meaning they adjusted somewhat well to well (See figure 5). More students who have attended sleepaway camp chose a 5, meaning very well, and more students who have not attended sleepaway camp, chose a 1, meaning not well. The average Likert score of those attending sleepaway camp was a 3.956, while those not attending had an average score of 3.705, indicating a slightly higher positive adjustment for those who attended summer camp.

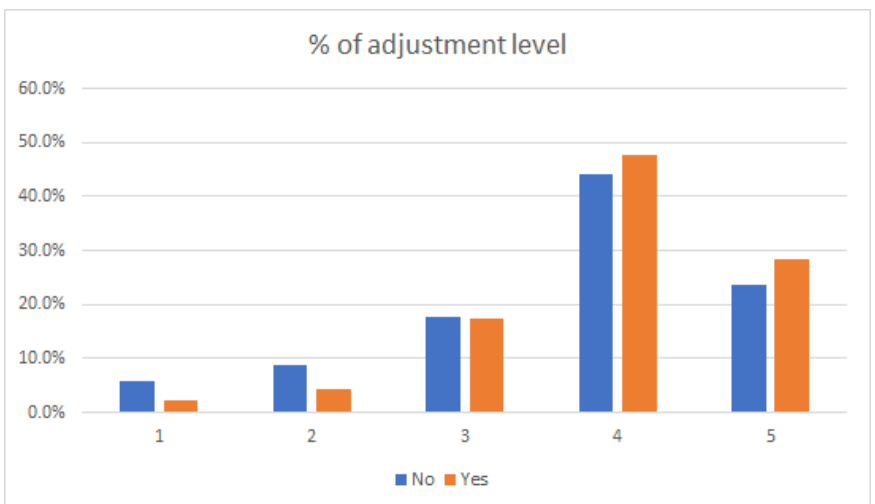

Figure 5. Graph indicating how well the participant believes they adjusted to college life in their first semester.

As for adjustment to living with a roommate, figure 6 shows a difference between those who have attended sleepaway camp versus those who have not. The majority of students who attended sleepaway camp rated living with a roommate as a 5, meaning they adjusted very well, whereas the majority of students who have not attended sleepaway camp rated their adjustment to a roommate as a 4, meaning they adjusted well. This shows a slight difference in how attending sleepaway camp can positively affect living with a roommate. 


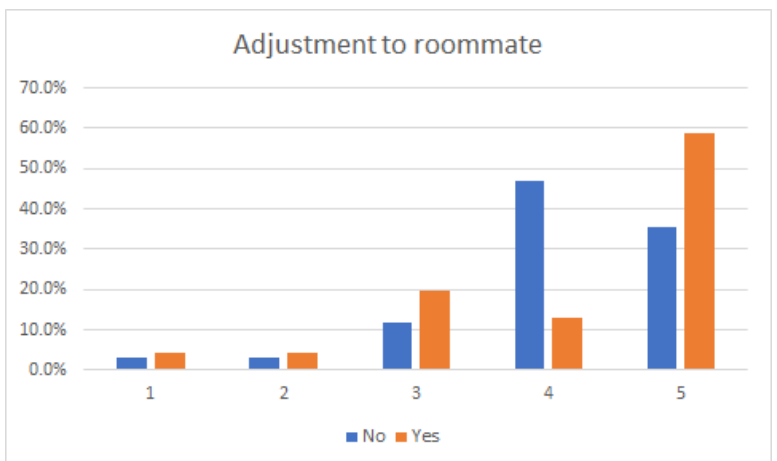

Figure 6. Graph indicating how well the participant adjusted to living with a roommate in the first semester.

\section{Camp Experience}

Participants who answered "yes" to having attended sleepaway camp before the age of 16 were then asked a set of questions about their camp experience. This allowed the researcher to analyze factors related to camp experiences and levels of homesickness in college. Figure 7 shows that most participants who had attended sleepaway camp before the age of 16 , had attended for six or more summers.

\section{If yes, how many summers did you attend sleep away camp?}

46 responses
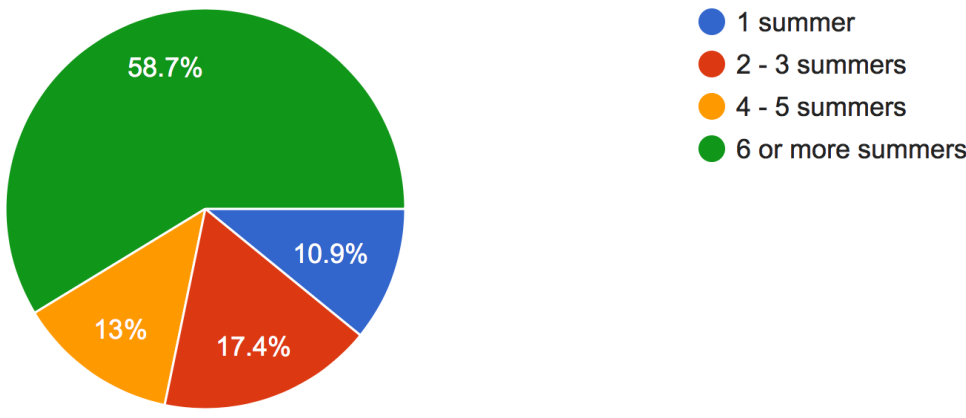

Figure 7. Graph indicating how many summers the participant went to sleepaway camp.

Table 1 shows the comparison of the number of years in camp and levels of homesickness. Out of the 27 respondents who went to sleepaway camp for six or more summers, 14, roughly half, rated their homesickness levels either a 4 or a 5, meaning they did not feel very homesick in their first semester. Of the $10.9 \%$ of students who attended sleepaway camp for only one summer, $60 \%$ rated their homesickness a 2 (homesick) and none rated their homesickness a 5 (not at all homesick). It is possible that these participants have a predisposition to homesickness, and that is a reason they only went to sleepaway camp for 1 summer. These results can show that attending camp for a more extended period of time affected how homesick the student felt. 
Table 1. Number of Years in Camp and Levels of Homesickness

\begin{tabular}{|c|c|c|c|c|c|}
\hline & 1 & 2 & 3 & 4 & 5 \\
\hline 1 year & $0 \%$ & $60 \%$ & $20 \%$ & $20 \%$ & $0 \%$ \\
\hline $2-3$ years & $12.50 \%$ & $12.50 \%$ & $37.50 \%$ & $12.50 \%$ & $25 \%$ \\
\hline $4-5$ years & $0 \%$ & $16.67 \%$ & $0 \%$ & $50 \%$ & $33.33 \%$ \\
\hline $6+$ years & $3.70 \%$ & $25.93 \%$ & $18.52 \%$ & $29.63 \%$ & $22.22 \%$ \\
\hline
\end{tabular}

Figure 8 shows the results of the question that asked about the length of time of the sleepaway camp. The researcher found that $30.4 \%$, or the majority of the participants' sleepaway camp, ranged from 3-4 weeks. The data was grouped into two categories for length of stay at sleepaway camp. Group one was two weeks or less, and group two was three weeks or more.

If yes, how long was your sleep away camp?

46 responses

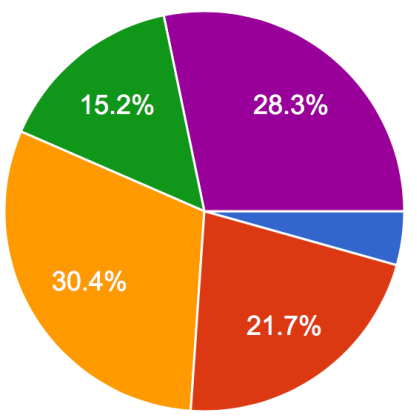

Figure 8. Graph indicating the length of the participants sleepaway camp

The data was analyzed in Table 2 to look at homesickness levels by these two categories. Participants with a shorter length of stay had a higher rating of homesickness $(33.33 \%)$ as compared with those who attended for longer stays (29.41\%). Participants with a longer stay had higher rates of not feeling homesick (52.94\% versus $41.67 \%)$. These results show that along with attending sleepaway camp for more summers, the length of the camp can affect homesickness levels as well.

Table 2. Length of Sleepaway Camp and Levels of Homesickness

\begin{tabular}{|c|c|c|}
\hline & 2 weeks or less & 3 weeks or more \\
\hline Not homesick (4 or 5 rating) & $41.67 \%$ & $52.94 \%$ \\
\hline Neutral homesick (3 rating) & $25 \%$ & $17.65 \%$ \\
\hline Homesick (1 or 2 rating) & $33.33 \%$ & $29.41 \%$ \\
\hline
\end{tabular}


A final "camp experience" question asked the participants to rate their overall camp experience on a scale of 1 to 5 , a 1 being the lowest and a 5 being the highest. Out of the 46 camp attending respondents, $82.6 \%$, or 38 , rated their camp experience as a 5, meaning they had an extremely positive camp experience. (See figure 9) No participants rated their camp experience as a 1 , but two rated their experience as a 2 . These two respondents both rated their homesickness levels as a 3, meaning they felt neutral. This can show that camp experience does not necessarily have an effect on homesickness levels.

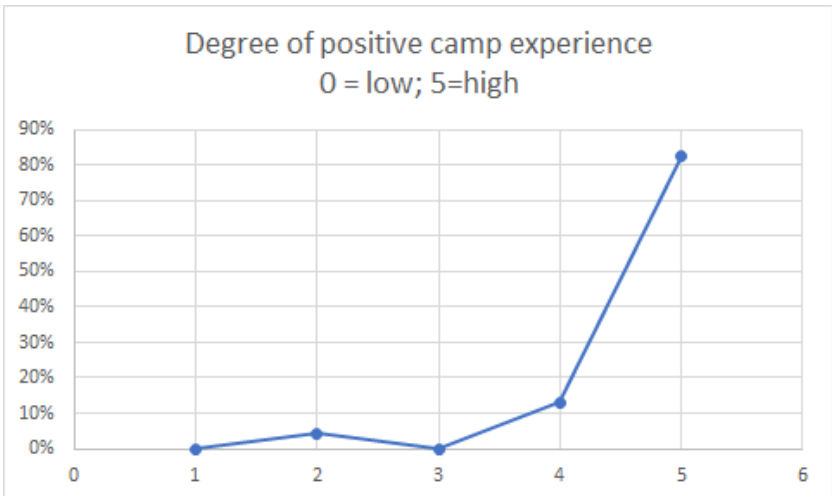

Figure 9. Graph indicating the participants degree of positive camp experience.

\section{Participants Opinions}

The final question was open-ended and asked, "Based on your experience, do you believe sleep away camp prepared you to adjust to college? Why or why not?" This question gave the participants the ability to share their thoughts on this question and give the researcher more in-depth results that were not just quantitative. After reading all responses, the researcher gathered that most participants felt that going to sleepaway camp has helped them in college. The most common phrases the researcher found in the answers were "made me more independent" and "learned how to live with others" and "learned how to live without my parents." For example, one respondent stated "Yes I learned how to be away from home and how to adapt to new settings." These 3 phrases were repeated over multiple answers, indicating that participants who attended sleepaway camp perceived the experience to help them in college.

Another category of responses stated that going to sleepaway camp did not help them in college because the experiences are different. One participant stated, "No, college has a lot of factors such as new financial responsibility and other stresses that summer camps did not provide." Several participants who did not attend sleepaway stated that if they did go, they believed it would help them in college. One participant stated, "I didn't go to sleep away camp, but I think it would have prepared me and my parents better."

\section{General Discussion}

It was hypothesized that homesickness in first-year college students would be lower in those who have attended summer camp versus those who have not. However, the data showed a small relationship between attending sleepaway camp and homesickness levels in first-year college students. Those who attended sleepaway camps for more years and a longer session did show to be less homesick than those who only went for one or two years for shorter sessions. This was a small subgroup of the study participants but did show a difference in results. Even though there were results that indicated attending sleepaway camp had a positive effect on "college experience," there were very few that suggested a relationship between homesickness levels and attending sleepaway camp. Thus, despite the fact that attending 
sleepaway camp before the age of 16 can positively affect other aspects of the college experience, such as living with a roommate, this study does not suggest that it has a high effect on homesickness levels.

\section{Limitations}

There are specific limitations of this study that may have affected the final research goal. First, the study's population only consisted of first-year students at the University of Florida, thus the results cannot be generalized to all college students. Although the sample size of this study was reliable, there was a discrepancy between the number of participants who went to sleepaway camp versus participants that did not. Out of the 80 participants, $57.5 \%$ (46) of the participants attended sleepaway camp before the age of 16 , while $42.5 \%$ (34) did not attend sleepaway camp before the age of 16. Even though the survey was given out randomly, it would have been more effective if there were an even number of participants who went to sleepaway camp and did not go to sleepaway camp. Another limitation the researcher came across was the accuracy of who was taking the survey. Although the first question asked the respondent to verify that they were a first-year student at the University of Florida, there was no way the researcher could verify that they did in fact meet the standards to take this survey. Students who took this survey had varying personalities, which acts as a limitation to the research. If a student is more outgoing, they may have had an easier time adjusting to college, versus a more introverted student, who may have struggled. These serve as limitations since they can affect college experience as well as homesickness levels. A limitation of this research was this survey did not account for in-state students versus out of state students. This could affect how many times the participant went home due to the ease of travel, as well as their levels of homesickness, as they can be farther away from home making them more homesick. A final limitation of this research is that it did not account for other summer programs, such as studying in another country or traveling with a group. These programs could also influence homesickness levels and acclimation to college.

\section{Significance of the Study}

The findings of this study are significant because they help point out a possible positive impact that attending sleepaway camp has on the campers. The results are especially important to those who attend sleepaway camp and are in college or plan to be because it can help them realize that they can utilize their camp experience in college. The results of this study are also significant to parents who are thinking about sending their child to a sleepaway camp. These results can help guide their decision if they believe that sending their child to sleepaway camp will have a positive impact on their college life in the future. Lastly, these results only add to the field of sleepaway camp research. The results show more positive effects of sleepaway camp in a place that has never been researched and can advance the research that has already been done in the field.

\section{Conclusion}

The results of this study show that attending sleepaway camp has minimal effect on homesickness levels in college, but does have a positive impact on the college experience as a whole. The results of the study show that the majority of participants, both those who have been to sleepaway camp and those who have not, rated their homesickness levels a 4, meaning "not homesick." Participants reported the frequency of calling and visiting home equally whether or not they attended sleepaway camp. This shows that sleepaway camp does not have a direct effect on homesickness levels.

The results do show that attending sleepaway camp has a positive impact on the "college experience." The average rating for adjustment to college as a whole for those who had attended sleepaway camp was a 3.956 while those who did not attend had an average score of 3.705. Going to sleepaway camp also showed a positive impact on 
living with a roommate, as the average rating for those who attended sleepaway camp was 4.173 , and the average rating for those who did not attend sleepaway camp was 4.088 .

As the research compiled during the literature review shows, attending sleepaway camp has shown to have a positive impact on social skills in teens. For example, the American Camp Association's "Five Year Camp Impact Study" (2005), showed a growth in 10 social skills from pre-camp to post-camp. Other research has shown that sleepaway camp and college show many parallels, which can help college students as well. One college student explained that going to sleepaway camp helped her in college by making it easier to live with a roommate and sharing spaces with others (Olsen, 2015). The evidence gathered throughout the literature review proved a distinct gap between attending sleepaway camp before the age of 16 and homesickness levels in first-year college students. This research did not confirm results found in previous literature on the subject of homesickness, but supported prior findings related to attending sleepaway camp and an overall positive college experience.

The research could be further studied to examine if those who attend sleepaway camp for 3-week sessions have a different acclimation experience to college than those who went for 8-week sessions. This can also be done by looking at how many years they attended sleepaway camp, such as looking at the results for people who attended four years or less, compared to those who attend for five years or more. Another further study could ask participants if they only went for one summer why they didn't go back or add a question to this study asking if the participant was homesick at camp. This method pertained to attending sleepaway camp as a whole, but looking more specifically at sleepaway camp length of years attended could find a further connection between the two. However, this research still justifies that attending sleepaway camp does have a minimal effect on homesickness levels in first-year students at the University of Florida, and a high positive impact on the college experience as a whole.

\section{References}

American Camp Association. (2005). Directions: Youth development outcomes of the camp experience. American Camp Association. Retrieved from https://www.acacamps.org/resource-library/research/directions-youth-development-outcomes-camp-experience

Bajrovic, L., Baker, E., Markel, M., Oosting, A., \& Hollingsworth, B. (n.d.). How to Deal with Homesickness in College. Retrieved from https://www.hap.org/blog/2018/09/homesick-in-college

Baskin, S. (2013, January 23). Unplugging our kids: Steve Baskin at TEDxSanAntonio [Video file]. Retrieved from http://www.youtube.com/watch?v=4rI3olRHxP4

Broussard, L., \& Meaux, J. (2007). Camp nursing: Rewards and challenges. Pediatric Nursing, 33(3), 238-42. Retrieved from https://www.ncbi.nlm.nih.gov/pubmed/17708183

Cohen, M. (2014, April 8). The social and emotional long-term benefits of summer camp. DNAInfo. Retrieved from https://www.dnainfo.com/new-york/20140408/lower-east-side/social-emotional-long-term-benefits-of-summercamp/

Diamond, E. (2015, July 8). Sleep away camp is good for kids and parents. HuffPost. Retrieved from http://www.huffpost.com/entry/sleepaway-camp-is-good-for-kids-and-parents_b_7705960

Esposito, Lisa. (2017, October 6). 7 signs of campus homesickness. US News. Retrieved from https://health.usnews.com/wellness/family/slideshows/7-signs-of-campus-homesickness?slide=2 
Khademi, A., \& Aghdam, A. A. F. (2013). The role of personality traits and resilience on homesickness of college students. Social and Behavioral Sciences, 82(2013), 537-541. doi:10.1016/j.sbspro.2013.06.305

Livingston, A. (2012, September 28). Homesickness common among new college students. Press-Republican. Retrieved from https://www.pressrepublican.com/news/local_news/homesickness-common-among-new-college-students/article_fd82cf59-0846-56a6-9ef3-583936e1c849.html

Meltzer, L. J., \& Rourke, M. T. (2005). Oncology summer camp: Benefits of social comparison. Children's Health Care, 34(4), 305-314. doi: 10.1207/s15326888chc3404_5

Monke, A. (2018, December 5). What's the best age for camp? American Camp Association. Retrieved from https://www.acacamps.org/campers-families/parent-blog/whats-best-age-camp.

Olsen, L. K. P., Powell, G. M., Garst, B. A., \& Bixler, R. D. Camp and college parallels: crucibles for transitionlinked turning-points. Journal of Youth and Development, 13(1). doi:10.5195/jyd.2018.558

Petrzela, N. M. (2017, August 7). Why fear of big cities led to the creation of summer camps. History. Retrieved from https://www.history.com/news/why-fear-of-big-cities-led-to-the-creation-of-summer-camps.

Rampton, J. (2017, November 6). Stepping away from technology is possible. research says it's needed to recharge your motivation. Inc. Retrieved from https://www.inc.com/john-rampton/stepping-away-from-technology-is-possible-research-says-its-needed-to-recharge-your-motivation.html.

Thompson, M. (2018, November 27). Research finds children learn social skills at camp. Retrieved from https://goldarrowcamp.com/research-finds-children-learn-social-skills-at-camp/.

Urani, M. A., Miller, S. A., Johnson, J. E., \& Petzel, T. P. (2003). Homesickness in socially anxious first year college students. College Student Journal, 37(3), 392-399. 